\title{
The Thermal and Photochemistry of a Pyrene Dihydrodioxin (PDHD) and Its Radical Cation: A Photoactivated Masking Group for ortho- Quinones
}

Eric T. Mack, A. Bjorn Carle, J. T.-M. Liang, W. Coyle, and R. Marshall Wilson* Department of Chemistry, University of Cincinnati, Cincinnati, OH 45221-0172.

\section{Supporting Information}

\section{Preparation of 10,10- Bis-(4 methoxy-phenyl)- 10,11-dihydro-9,12-dioxa-} benzo[e]pyrene (1).

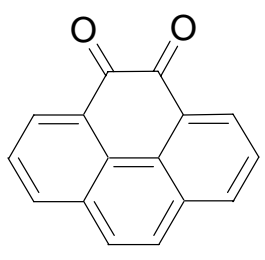

3
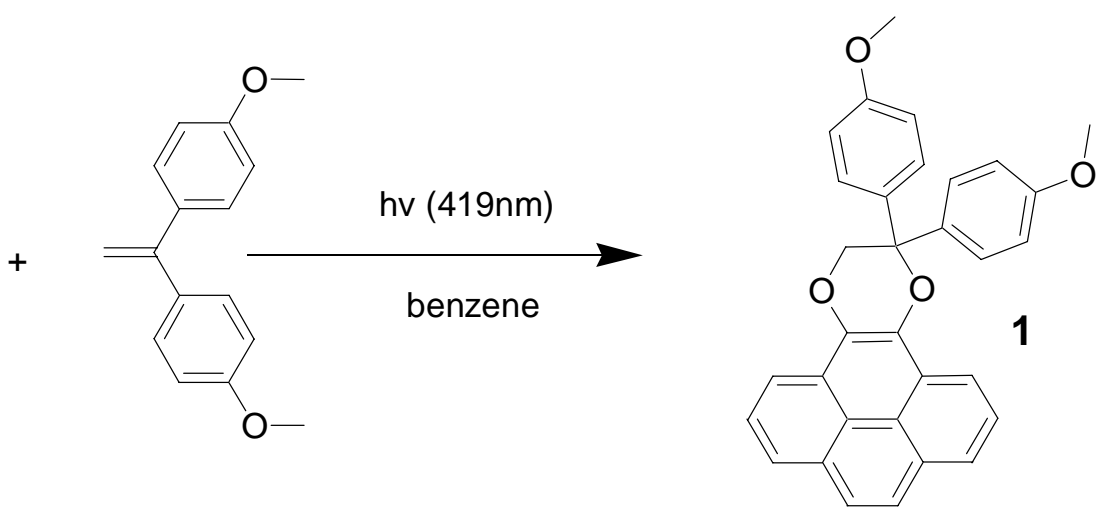

A solution of $0.5 \mathrm{~g}(2.153 \mathrm{mmol})$ of pyrene-4,5-dione and $1.04 \mathrm{~g}(4.31 \mathrm{mmol})$ of 1,1-di (4-methoxyphenyl)ethylene in $250 \mathrm{~mL}$ of benzene was placed in a $250 \mathrm{~mL}$ photoreactor flask. The solution was heated to dissolve the quinone then degassed by sparging with Ar for 5 minutes. The flask was placed under a positive pressure of $\mathrm{Ar}$ and cooled to $15^{0} \mathrm{C}$ while irradiated in a Rayonet photoreactor equipped with $419 \mathrm{~nm}$ bulbs for a total of 16.75 hrs. The benzene was removed under reduced pressure in the presence of added silica gel. The reddish brown silica gel was suspended in $30 \mathrm{~mL}$ of $10 \%$ EtOAc in hexanes and the suspension loaded onto a column of silica gel (60A finer than 230 mesh). 
The product was eluted with a gradient elution (10-20\% EtOAc in hexanes) to yield a viscous brown oil. The oil was crystallized from $\mathrm{CH}_{2} \mathrm{Cl}_{2}$ and hexanes and the resulting crystals recrystallized to obtain a crop of colorless polygons.

Product $\mathrm{C}_{32} \mathrm{H}_{24} \mathrm{O}_{4}$

Yield $0.1312 \mathrm{~g}, 12.9 \%$

${ }^{1} \mathbf{H}$ NMR $\left(\mathrm{CDCl}_{3}, 250 \mathrm{MHz}\right) \delta 8.63(\mathrm{~d}, J=7.4 \mathrm{~Hz}, 1 \mathrm{H}), 8.32(\mathrm{~d}, J=7.4 \mathrm{~Hz}, 1 \mathrm{H}), 8.04-$

${ }^{13} \mathbf{C ~ N M R}\left(\mathrm{CDCl}_{3}\right) \delta 159.2,133.8,133.3,130.9,130.8,128.2,128.2,127.2,126.2,125.9$, $125.7,125.4,123.6,121.1,121.0,118.0,117.9,113.8,19.4,55.1$

IR $\left(\mathrm{CCl}_{4}\right) v_{\max } 3054-2839,1610,1512,1312,1178,1113,1034 \mathrm{~cm}^{-1}$

HRMS (ESI) calcd for $\mathrm{C}_{32} \mathrm{H}_{24} \mathrm{O}_{4} \mathrm{Na}$ 473.1753; found, 473.1777

UV-Vis ( $\lambda$ max $2 \% \mathrm{CH}_{2} \mathrm{Cl}_{2}$ in hexanes $), \quad 291 \mathrm{~nm}\left(26,926 \mathrm{M}^{-1} \mathrm{~cm}^{-1}\right), 347 \mathrm{~nm}\left(12,817 \mathrm{M}^{-}\right.$ $\left.{ }^{1} \mathrm{~cm}^{-1}\right)$ 


\section{Preparation of 10, 10-Bis-[4-(3-bromo-propoxy)-phenyl]-10,11-dihydro-9,12-dioxa- benzo[e]pyrene}
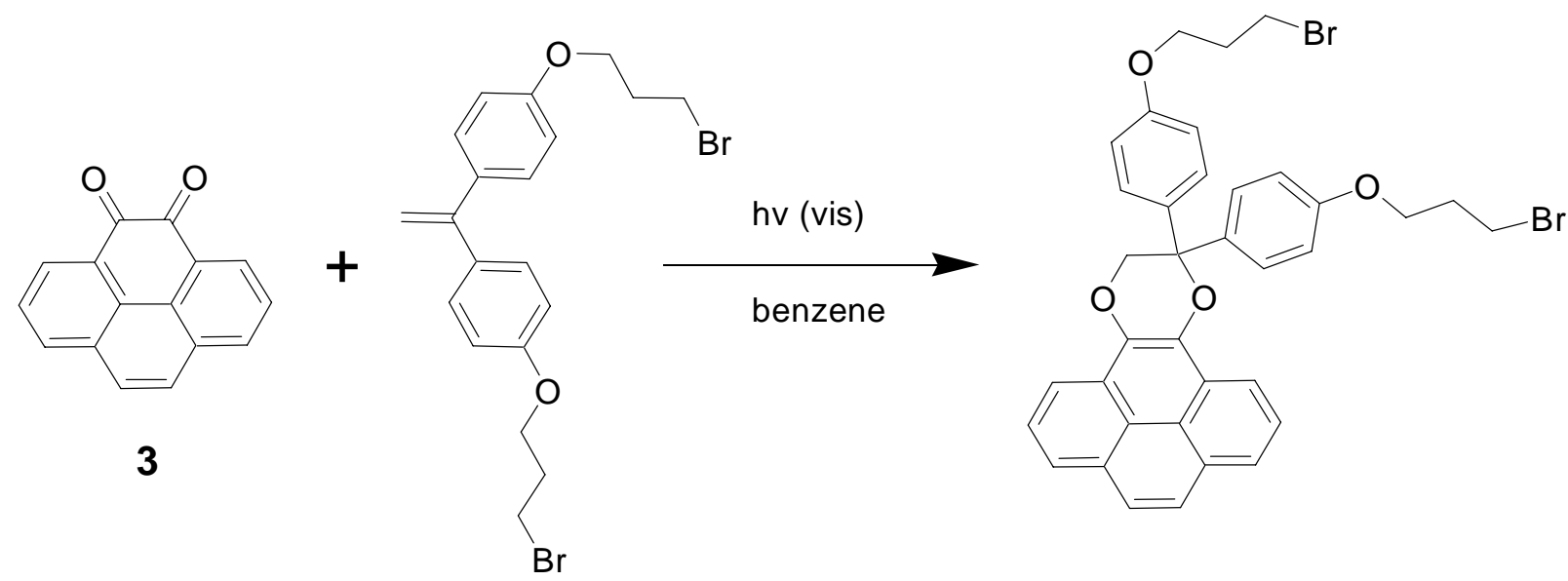

A solution of 0.141 grams $(0.31 \mathrm{mmole})$ of 3-bromo-1-(4-\{1-[4-(3-

bromoproproxy)phenyl]vinyl $\}$ phenoxy)propane and 0.0.069 grams $(0.30 \mathrm{mmole})$ of pyrene-4,5-dione $(3)$ in $150 \mathrm{~mL}$ of benzene was prepared in a $250 \mathrm{~mL}$ pyrex round bottom flask. The solution was heated to dissolve the quinone then degassed by sparging with $\mathrm{Ar}$ for 15 minutes. The flask under a positive pressure of Argon and cooled to $7^{0} \mathrm{C}$ while irradiating with the visible lines of an argon ion laser. The benzene was removed under reduced pressure and the resulting thick black residue was purified by rotary chromatography using a gradient elution ( 0-10\% EtOAc in hexanes). The product was collected as bright blue fluorescent band (254 nm irradiation). The resulting oil was crystallized from $\mathrm{CCl}_{4}$ and hexanes.

Product $\quad \mathrm{C}_{36} \mathrm{H}_{30} \mathrm{O}_{4} \mathrm{Br}_{2}$

Yield $\quad 0.0389 \mathrm{~g}$ (0.057 mmole) $18 \%$
Mol. Weight $686.43 \mathrm{~g} / \mathrm{mol}$

Melting point $140-144^{\circ} \mathrm{C}$ 
${ }^{1} \mathbf{H} \mathbf{N M R}\left(\mathrm{CDCl}_{3}\right) \delta 8.64(\mathrm{dd}, J=1.6 \mathrm{~Hz}, J=7.0 \mathrm{~Hz}, 1 \mathrm{H}), 8.34(\mathrm{dd}, J=1.0 \mathrm{~Hz}, J=7.5$ Hz, 1H), 8.09-7.89 (m, 6H), $7.48(\mathrm{~d}, J=11.8 \mathrm{~Hz}, 4 \mathrm{H}), 6.77(\mathrm{~d}, J=8.7 \mathrm{~Hz}, 4 \mathrm{H}), 4.85(\mathrm{~s}$, 2H), $3.94(\mathrm{t}, J=5.8 \mathrm{~Hz}, 4 \mathrm{H}), 3.49(\mathrm{t}, J=6.3 \mathrm{~Hz}, 4 \mathrm{H}), 2.19(\mathrm{~m}, 4 \mathrm{H})$

${ }^{13} \mathbf{C} \mathbf{N M R}\left(\mathrm{CDCl}_{3}\right) \quad 158.3,133.7,133.5,133.3,130.8,130.8,128.2,127.2,126.2,125.9$, $125.7,125.3,123.6,121.0,121.0,118.0,117.8,114.3,79.4,70.5,65.1,32.2,29.9$ IR $\left(\mathrm{CCl}_{4}\right) v_{\max } 3054,2931,1610,1511,1313,1246,1177,1112,1034 \mathrm{~cm}^{-1}$ HRMS (ESI) calcd for $\mathrm{C}_{36} \mathrm{H}_{30} \mathrm{O}_{4} \mathrm{Br}_{2} \mathrm{~K}$ 725.0128; found, 725.0126 UV-Vis $\left(\lambda \max \mathrm{CH}_{2} \mathrm{Cl}_{2}\right), 349 \mathrm{~nm}\left(14,000 \mathrm{M}^{-1} \mathrm{~cm}^{-1}\right)$

Preparation of [3-(4-\{10-[4-(3-trimethylamino-propoxy)-phenyl]10,11-dihydro-9,12dioxa-benzo[e]pyren-10yl\}-phenoxy)-propyl]-trimethyl amine dichloride salt (2)

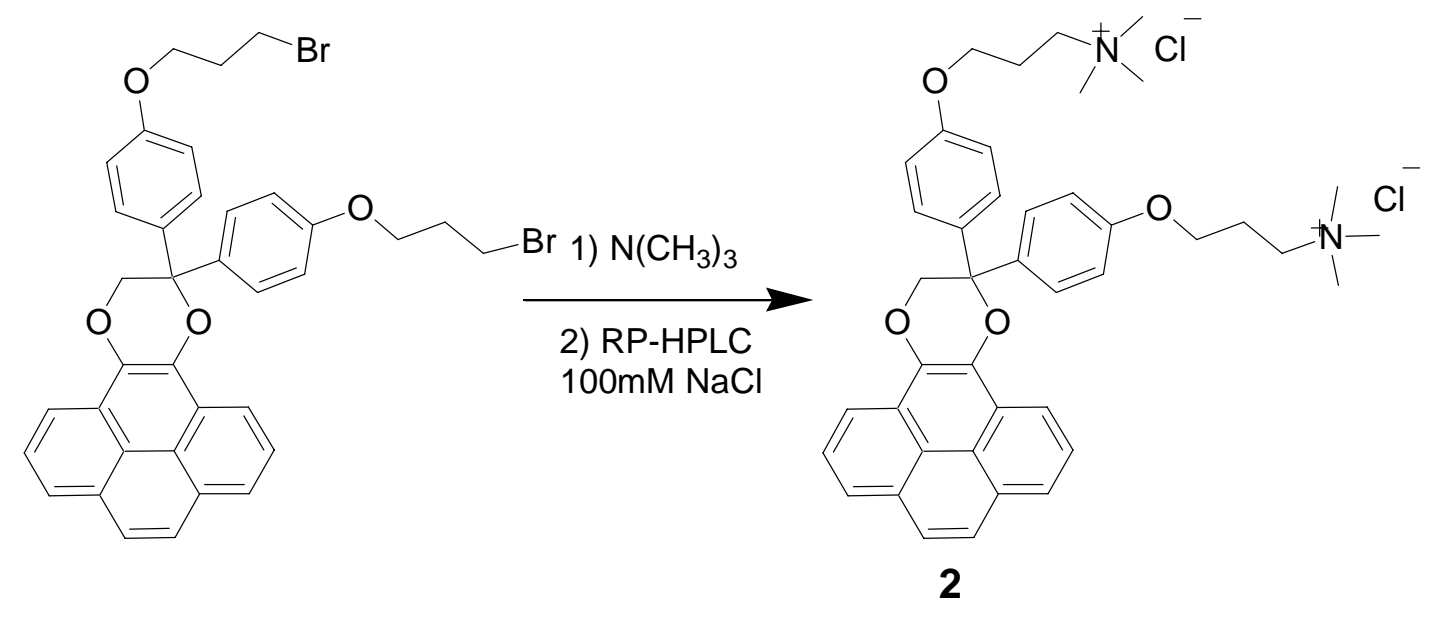

$\mathrm{A} \mathrm{CDCl}_{3}$ 0.051g (0.074 mmole) of 10, 10-Bis-[4-(3-bromo-propoxy)-phenyl]-10,11dihydro-9,12-dioxa-benzo[e]pyrene was prepared in a sealable vial. Anhydrous trimethylamine $(5 \mathrm{~mL})$ was condensed into the vial which was then sealed tightly. After $24 \mathrm{hrs}$ the precipitate was collected by filtration and washed three times with room temperature ether. The resulting solid was purified by preparative reverse phase HPLC 
using a gradient elution of $65-90 \% \mathrm{ACN}$ in $100 \mathrm{mM} \mathrm{NaCl}$. The solvent was removed by a combination of rotary evaporation and freeze drying. The product was extracted from the resulting salt residue by washing with $3 \times 20 \mathrm{~mL}$ portions of dry ACN to yield a hard white solid after removal of the solvent.

Product $\mathrm{C}_{42} \mathrm{H}_{48} \mathrm{O}_{4} \mathrm{~N}_{2} \mathrm{Br}_{2}$

Yield $0.047 \mathrm{~g}, 91.2 \%$
Mol. Weight $804.65 \mathrm{~g} / \mathrm{mol}$

Melting point decomposes $230-250^{\circ} \mathrm{C}$

${ }^{1} \mathbf{H}$ NMR $\left(\mathrm{CD}_{2} \mathrm{Cl}_{2}-\mathrm{CD}_{3} \mathrm{OD}, 250 \mathrm{MHz}\right) \delta 8.61(\mathrm{dd}, J=1.2 \mathrm{~Hz}, J=7.9 \mathrm{~Hz}, 1 \mathrm{H}), 8.28(\mathrm{~d}, J$ $=8.0 \mathrm{~Hz}, 1 \mathrm{H}), 8.08-7.88(\mathrm{~m}, 6 \mathrm{H}), 7.52(\mathrm{~d}, J=8.8 \mathrm{~Hz}, 4 \mathrm{H}), 6.84(\mathrm{~d}, J=8.8 \mathrm{~Hz}, 4 \mathrm{H}), 4.90$ (s, 2H), $3.99(\mathrm{t}, J=5.5 \mathrm{~Hz}, 4 \mathrm{H}), 3.47(\mathrm{~m}, 4 \mathrm{H}), 3.11(\mathrm{~s}, 18 \mathrm{H}), 2.15(\mathrm{~m}, 4 \mathrm{H})$ ${ }^{13} \mathbf{C ~ N M R}\left(\mathrm{CDCl}_{3}\right) \delta 158.9,134.9,131.8,128.9,128.0,126.8,126.6,124.4,115.1,80.2$, $71.2,65.3,65.0,65.0,23.9$ IR $\left(\mathrm{CCl}_{4}\right)$ 3422(s and broad, $\left.\mathrm{H}_{2} \mathrm{O}\right), 2913(\mathrm{w}), 2868(\mathrm{w}), 1630(\mathrm{~m}), 1608(\mathrm{~m}), 1511(\mathrm{~m})$, 1475(m), 1385(s), 1313(m), 1246(m), 1174(m), 1111(m), $1050(\mathrm{~m}), 833(\mathrm{~m}), 716(\mathrm{w}) \mathrm{cm}^{-1}$ HRMS (ESI) calcd for $\mathrm{C}_{42} \mathrm{H}_{48} \mathrm{O}_{4} \mathrm{~N}_{2}{ }^{2+} 322.1807$; found, 322.1803 UV-Vis $(\lambda \max , 10 \mathrm{mM}$ phosphate $10 \mathrm{mM} \mathrm{NaCl}$ buffer $\mathrm{pH} 7.2) 243 \mathrm{~nm}(36,942 \pm 1,526$ $\left.\mathrm{M}^{-1} \mathrm{~cm}^{-1}\right), 288 \mathrm{~nm}\left(14,032 \pm 231 \mathrm{M}^{-1} \mathrm{~cm}^{-1}\right), 349 \mathrm{~nm}\left(9,142 \pm 70 \mathrm{M}^{-1} \mathrm{~cm}^{-1}\right), 353 \mathrm{~nm}(9,345 \pm$ $\left.68 \mathrm{M}^{-1} \mathrm{~cm}^{-1}\right)$ 